14,04

\title{
Старение и разрушение (пробой) полимерных пленок в переменном электрическом поле
}

\author{
(C) В.А. Закревский ${ }^{1}$, В.А. Пахотин ${ }^{1}$, Н.T. Сударь ${ }^{2}$ \\ ${ }^{1}$ Физико-технический институт им. А.Ф. Иофрфе РАН, \\ Санкт-Петербург, Россия \\ ${ }^{2}$ Санкт-Петербургский политехнический университет Петра Великого, \\ Санкт-Петербург, Россия \\ E-mail: v.pakhotin@mail.ioffe.ru
}

Поступила в Редакцию 3 апреля 2019 г.

В окончательной редакции 3 апреля 2019 г.

Принята к публикации 23 мая 2019 г.

Предложено объяснение различия электропрочностных свойств полимеров в постоянном и переменном электрическом полях. Выделение энергии при рекомбинации инжектированных в полимерный диэлектрик электронов и дырок рассматривается как фактор, ускоряющий процессы электрического старения этих диэлектриков в переменном электрическом поле.

Показано, что безызлучательная релаксация электронных возбужденных состояний, вызывающая разрывы связей в макромолекулах и образование свободных радикалов, приводит к образованию в полимерном диэлектрике глубоких электронных ловушек, в результате чего ускоряется ионизация макромолекул в электрическом поле за счет переходов электронов в эти ловушки. В твердотельной плазме возникает эффект экранирования, приводящий к снижению потенциала ионизации молекул. В результате увеличивается скорость ионизации макромолекул, то есть скорость образования носителей заряда, что приводит к снижению электрической долговечности полимерного диэлектрика в переменном электрическом поле по сравнению с долговечностью полимеров в постоянном поле.

Ключевые слова: полимер, электрический пробой, электролюминесценция, потенциал ионизации молекул.

DOI: 10.21883/FTT.2019.10.48276.445

\section{1. Введение}

Полимеры широко используются в качестве изоляционных материалов. Поэтому исследование их электропрочностных свойств является актуальной задачей. Несмотря на то, что изучение воздействия сильных электрических полей на диэлектрики продолжается много десятилетий [1], общепринятая точка зрения на природу электрической прочности полимеров отсутствует.

Многие авторы полагают, что причиной пробоя полимерной изоляции является образование под действием электрического поля в полимере полостей микронных размеров, в которых могут развиваться газовые разряды. Отметим, что газовые разряды в полостях с размерами меньшими $5 \mu \mathrm{m}$ не развиваются в полях, обычно используемых в опытах по изучению долговечности полимеров, то есть в полях с напряженностью $F \sim 10^{6} \mathrm{~V} / \mathrm{cm}[1,2]$. Считается, что полости появляются в результате разрыва макромолекул [3-5]. При этом не учитывается, что прочность межатомных химических связей в макромолекуле намного превосходит напряжения $\sigma$, которые могут действовать в диэлектриках в электрическом поле. Действительно, $\sigma=\varepsilon \varepsilon_{0} F^{2} / 2$, где $\varepsilon-$ диэлектрическая проницаемость полимера, $\varepsilon_{0}$ - электрическая постоянная, так что даже при $F=10^{7} \mathrm{~V} / \mathrm{cm}, \sigma=1 \mathrm{~kg} / \mathrm{mm}^{2}$, в то время как прочность химических связей составляет $\sim 10^{3} \mathrm{~kg} / \mathrm{mm}^{2}$ [6]. К тому же разрывы макромолекул не могут привести к образованию полости, поскольку плотность материала при разрыве межатомных связей не меняется.

В качестве аргумента в пользу предположения об образовании в полимерах пор под воздействием электрического поля иногда используется тот факт, что механическая прочность некоторых аморфных полимеров невелика и составляет всего несколько $\mathrm{kg} / \mathrm{mm}^{2}$ [7]. Такой подход представляется несостоятельным, поскольку в механическом поле макромолекулы разрываются вследствие возникновение больших перенапряжений, вызванных неоднородностью структуры полимеров.

Разрушение полимеров в электрическом поле нельзя также связывать с возникновением электронных лавин, поскольку в условиях прыжкового транспорта, характерного для органических соединений, длина свободного пробега, равная межмолекулярному расстоянию, не превышает $1 \mathrm{~nm}$, что недостаточно для набора электронами энергии, необходимой для ионизации молекул.

Недавно был предложен так называемый ионизационный механизм электрического разрушения полимеров, с единых позиций описывающий их старение и пробой вследствие быстрого роста концентрации носителей заряда $[8,9]$. Разрушение полимеров в электрическом поле в работах $[8,9]$ рассматривается как следствие накопления квазисвободных зарядов в результате туннельной ионизации макромолекул в сильном электрическом поле. 
При накоплении в полимере разноименных зарядов возникает эффект экранирования, что приводит к снижению потенциала ионизации молекул и, следовательно, к ускорению их ионизации. Чем выше концентрация носителей заряда, тем выше скорость ионизации и скорость накопления зарядов. Протекание этого самоускоряющегося процесса (процесса с положительной обратной связью), то есть процесса, скорость которого возрастает со временем, приводит на определенном этапе (при достижении критической концентрации зарядов) к резкому изменению свойств полимера - к его пробою.

Эта ситуация подобна той, которая реализуется при термической ионизации атомов и молекул, описываемой формулой Саха. Термоионизация также резко ускоряется при накоплении зарядов [10].

В работах $[8,9]$ отмечается, что в ионизированных макромолекулах (положительных макроионах) понижена энергия разрыва химических связей. Она снижается также под действием электрического поля. Поэтому в электрическом поле происходит разрыв макромолекул с образованием химически активных свободных макрорадикалов, вступающих в химические реакции с соседними макромолекулами, в результате чего в полимерах накапливаются новые атомные группировки (химические дефекты в макромолекулах с положительным сродством к электрону), являющиеся ловушками электронов. Появление ловушек ускоряет туннельную ионизацию молекул в электрическом поле из-за сужения потенциального барьера.

Выполненные на основе этих представлений оценки долговечности полимерных материалов в постоянном электрическом поле различной напряженности находятся в удовлетворительном согласии с экспериментальными данными [9]. Однако полимерная изоляция в основном используется в переменном поле. Вместе с тем хорошо известно, что электрическая прочность полимерных диэлектриков в переменном электрическом поле существенно ниже, чем в постоянном [11] и, в частности, известно, что долговечность полимеров в переменном поле примерно на два порядка величины меньше долговечности в постоянном поле [12,13]. Этот факт прямо указывает на то, что дефектообразование в полимерах под действием переменного поля происходит гораздо более интенсивно, чем под действием постоянного поля. Следовательно при рассмотрении разрушения полимеров в переменном электрическом поле необходимо дополнительно учитывать процессы, интенсивно протекающие только в переменном поле.

Поэтому настоящая работа посвящена выяснению вопроса о том, возможно ли использовать представления об ионизационном механизме пробоя для описания разрушения полимеров в переменном электрическом поле.

\section{2. Постановка задачи}

Полагалось, что на макромолекулы полимерного диэлектрика воздействует переменное электрическое поле, напряженность которого изменяется во времени как $F(t)=F_{0} \sin (2 \pi f t)$. Здесь $F_{0}-$ амплитуда напряженности поля, действующего на макромолекулу, $f-$ его частота. В переменном электрическом поле в полимерах возникает интенсивная электролюминесценция (ЭЛ). В постоянном электрическом поле ее интенсивность невелика. В полимерах ЭЛ возникает в результате рекомбинации электронов с положительными молекулярными ионами (дырками) [14-17]. Благоприятные условия для рекомбинации разноименных зарядов в переменном поле создаются из-за того, что из одного и того же электрода в разные полупериоды инжектируются и электроны и дырки. Рекомбинация при этом происходит в узком приэлектродном слое полимера толщиной

$$
d_{0}=\int_{0}^{T / 2} \mu F_{0} \sin (2 \pi f t) d t=\frac{\mu F_{0}}{\pi f},
$$

где $\mu$ - подвижность инжектируемых носителей заряда, $T=1 / f$.

В настоящей работе предполагается рассмотреть электронно-дырочную рекомбинацию как фактор, ускоряющий процессы электрического старения полимеров в переменном поле и с учетом этого обстоятельства оценить возможное снижение электрической долговечности (времени жизни в электрическом поле) полимерного диэлектрика в переменном поле по сравнению с постоянным. Отметим, что на связь старения полимеров в электрическом поле с ЭЛ ранее указывалось в работах $[18-20]$.

Испускание света в видимом диапазоне является следствием релаксации возбужденных состояний, возникающих при электронно-дырочной рекомбинации. Электронные возбуждения молекул возникают, поскольку энергия $\Delta E$, выделяемая при рекомбинации зарядов, достаточно велика. Ее величина равна [21]:

$$
\Delta E \approx I_{G}-A_{G}-2 P-U_{c},
$$

где $I_{G}$ и $A_{G}$ - энергия ионизации и сродство молекулы к электрону в газовой фазе, $P-$ энергия поляризации полимера единичным зарядом, $U_{c}$ - энергия кулоновского взаимодействия зарядов. По оценке [22] при рекомбинации зарядов в конденсированных органических соединениях выделяется энергия 4.5-7.5 eV. Очевидно, что эта энергия превосходит энергию возбуждения фотолюминесценции и энергию разрыва ковалентных химических связей.

Высшие возбужденные состояния дезактивируются за время $\sim 10^{-12} \mathrm{~s}$, поэтому все переходы в основные состояния осуществляются из первых возбужденных состояний. При рекомбинации зарядов в соответствие со спиновой статистикой синглетные и триплетные возбужденные состояния образуются в соотношении $1: 3$. Синглетные состояния релаксируют в основном вследствие излучательных переходов $S_{1} \rightarrow S_{0}$ (флюоресценция). Переходы из триплетных состояний в основное запрещены 
по спину. Поэтому дезактивация возбужденных триплетных состояний происходит, в основном, безызлучательно и их энергия расходуется на инициирование химических превращений, связанных с разрывами химических связей и образованием свободных радикалов. Соотношение $\varphi$ между числом химических превращений в молекулах (числом актов диссоциации межатомных химических связей) и числом актов рекомбинации определяется как отношение константы скорости диссоциации к сумме констант скоростей процессов дезактивации возбужденного триплетного состояния, то есть

$$
\varphi=\frac{k_{d}}{k_{d}+k_{p}+k_{T S}},
$$

где $k_{d}$ - константа скорости диссоциации межатомных связей (деструкции возбужденных макромолекул), $k_{p}$ константа скорости фосфоресценции, $k_{T S}$ - константа скорости интеркомбинационного перехода. Поскольку $k_{d} \gg k_{p}, k_{T S}[23]$, то $\varphi \approx 1$. Действительно, установлено, что деструкция макромолекул, возбужденных в триплетное состояние, происходит с вероятностью, близкой к единице [24].

Таким образом, в результате безызлучательной релаксации возбужденных триплетных состояний происходят разрывы химических связей в макромолекуле с образованием свободных радикалов (обрывков макромолекул с неспаренными электронами). Образование свободных радикалов в этих условиях наблюдалось в [24]. Протекание химических реакций, инициированных свободными радикалами, вызывает образование химических дефектов в макромолекулах, являющихся ловушками электронов. В полиэтилене это атомные группы с двойными $\mathrm{C}=\mathrm{C}$ и $\mathrm{C}=\mathrm{O}$-связями [25]. Образование электронных ловушек ускоряет ионизацию полимерных молекул в электрическом поле из-за сужения потенциального барьера, сквозь который туннелируют электроны $[8,9]$.

В дальнейшем предполагается, что концентрация ловушек $N_{t}$ равна концентрации свободных радикалов, то есть что каждый свободный радикал инициирует образование одной глубокой (химической) электронной ловушки. Ясно, что при рассмотрении воздействия переменного электрического поля на полимеры необходимо дополнительно учитывать разрывы химических связей в макромолекулах из-за выделения энергии при рекомбинации разноименных зарядов инжектированных из электродов (в разные полупериоды воздействующего напряжения). Скорость образования ловушек при рекомбинации зарядов, можно оценить на основании литературных данных, полученных при исследовании интенсивности ЭЛ полимеров в переменном электрическом поле. Эта скорость пропорциональна мгновенному значению яркости ЭЛ $J(t)$, то есть

$$
\frac{d N_{t}^{\prime \prime}}{d t}=\frac{J(t)}{\beta V_{r}},
$$

где $N_{t}^{\prime \prime}$ - концентрация ловушек, образующихся при рекомбинации зарядов, $\beta-$ коэффициент пропорциональности (внешний квантовый выход). Значение $\beta$ оценивается в пределах $10^{-6}-10^{-4}[18,22,26], V_{r}-$ объем области, из которой регистрируется свечение.

Известно, что под действием переменного электрического поля свечение в полимерах наблюдается каждый полупериод действующего напряжения. Характер изменения яркости ЭЛ в течение полупериода близок к синусоидальному, причем максимум свечения сдвинут относительно максимума приложенного напряжения на угол $\sim \pi / 4[14]$, что позволяет аппроксимировать $J(t)$ выражением

$$
J(t)=A \mid \sin \left(2 \pi f t-\frac{\pi}{4}\right),,
$$

где $A-$ нормировочный множитель.

Для определения нормировочного множителя $A$ можно использовать экспериментальные данные об интегральной яркости ЭЛ $J\left(F_{\mathrm{rms}}\right)$ (числа квантов испущенных за одну секунду) в зависимости от среднеквадратичного значения напряженности электрического поля. Величина $J\left(F_{\mathrm{rms}}\right)$ связана с $J(t)$ соотношением $\int_{0}^{1} J(t) d t=J\left(F_{\mathrm{rms}}\right)$. Полученные при исследовании ЭЛ полиэтилентерефталата в переменном поле $(f=50 \mathrm{~Hz})$ [27] данные о зависимости $J\left(F_{\mathrm{rms}}\right)$ позволяют аппроксимировать рассматриваемую зависимость при $F_{\mathrm{rms}} \geq 0.8 \mathrm{MV} / \mathrm{cm}$ соотношением

$$
J\left(F_{\mathrm{rms}}\right)=G \exp \left(\alpha F_{\mathrm{rms}}\right),
$$

где $G=5.4 \mathrm{~s}^{-1}, \alpha=1.14 \mathrm{~cm} / \mathrm{MV}$.

Здесь следует отметить, что вследствие наличия в полимере областей усиления поля используемое далее в расчетах значение амплитуды напряженности действующего переменного поля $F_{0}$ превышает значение амплитуды переменного электрического поля $F_{a}$, определяемого экспериментально и равного $F_{a}=U_{0} / d=\sqrt{2} F_{\mathrm{rms}}$ (если $U_{0}$ - амплитуда переменного напряжения, приложенного к образцу толщиной $d$ ). Поэтому $F_{0}=q U_{0} / d$, где $q$ - коэффициент электрических перенапряжений.

Таким образом, для описания процесса старения и разрушения полимеров в переменном электрическом поле, может быть использована следующая система кинетических уравнений

$$
\begin{gathered}
\frac{d}{d t} n_{\mathrm{HL}}^{+}=k_{\mathrm{HL}}\left(N_{0}-n_{\mathrm{HL}}^{+}-n_{\mathrm{HT}}^{+}\right)-k_{t f} n_{\mathrm{HL}}^{+}-k_{r} n_{\mathrm{HL}}^{+} n_{e}, \\
\frac{d}{d t} n_{\mathrm{HT}}^{+}=k_{\mathrm{HT}}\left(N_{t}-N_{t}^{\prime}\right)-k_{t f} n_{\mathrm{HT}}^{+}-k_{r} n_{\mathrm{HT}}^{+} n_{e}, \\
\frac{d}{d t} n_{r}^{+}=k_{t f}\left(n_{\mathrm{HL}}^{+}+n_{\mathrm{HT}}^{+}\right)-k_{r} n_{r}^{+} n_{e}, \\
\frac{d}{d t} N_{t}=k_{t f}\left(n_{\mathrm{HL}}^{+}+n_{\mathrm{HT}}^{+}\right)+\frac{J(t)}{\beta V_{r}}, \\
\frac{d}{d t} N_{t}^{\prime}=\left(k_{\mathrm{HT}}+\sigma_{t} \mu_{e} F n_{e}\right)\left(N_{t}-N_{t}^{\prime}\right)
\end{gathered}
$$

и уравнение электронейтральности плазмы, имеющее вид

$$
n_{\mathrm{HL}}^{+}+n_{\mathrm{HT}}^{+}+n_{r}^{+}-n_{e}-N_{t}^{\prime}=0 .
$$

Здесь $N_{0}$ - начальная концентрация способных ионизоваться фрагментов молекул (например, мономерных 
звеньев); $n_{\mathrm{HL}}^{+}$- концентрация дырок, образующихся при туннельном переходе электронов с верхней заполненной молекулярной орбитали (уровня) на низшую свободную орбиталь соседней молекулы (уровень LUMO) - переход HOMO-LUMO; $n_{\mathrm{HT}}^{+}$- концентрация дырок, образующихся при туннельных переходах электронов с уровня HOMO на глубокую ловушку (переход НОМО-TRАР); $n_{r}^{+}$- концентрация дырок, образующихся при термофлуктуационном распаде макроионов $n_{\mathrm{HL}}^{+}$и $n_{\mathrm{HT}}^{+} ; n_{e}-$ концентрация свободных электронов; $N_{t}-$ полная концентрация ловушек; $N_{t}^{\prime}-$ концентрация ловушек, занятых электронами; $\mu_{e}-$ подвижность электронов; $\sigma_{t}$ - сечение захвата электрона химической ловушкой; $F-$ напряженность электрического поля; $k_{r}-$ константа скорости бимолекулярной рекомбинации; $k_{t f}-$ константа скорости термофлуктуационного разрыва химических связей в положительных макроионах; $k_{\mathrm{HL}}$ и $k_{\mathrm{HT}}$ - константы скоростей туннельных электронных переходов HOMO-LUMO и HOMO-TRAP.

Уравнения (3) и (4) определяют скорость накопления дырок, возникающих в результате переходов HOMO-LUMO и HOMO-TRAP, как разницу между соответствующими скоростями туннельных переходов и скоростями термофлуктуационного распада макроионов и электронно-дырочной рекомбинации. Уравнение (5) определяет скорость накопления дырок, образующихся при термофлуктуационном распаде молекулярных ионов $n_{\mathrm{HL}}^{+}$и $n_{\mathrm{HT}}^{+}$и исчезающих при рекомбинации их со свободными электронами. При написании уравнения (6) предполагалось, что ловушечные состояния могут образовываться как за счет термофлуктуационного распада молекулярных ионов (первое слагаемое), так и за счет распада возбужденных молекул (второе слагаемое). При написании уравнения (7) учитывалось, что заполнение ловушечных состояний электронами возможно как за счет туннельных переходов HOMO-TRAР, так и за счет захвата на ловушки свободных электронов. При этом полагалось, что время освобождения электрона из глубокой ловушки превосходит время жизни полимера в электрическом поле.

Известно, что константа скорости термофлуктуационного разрыва химических связей в положительных макроионах $k_{t f}$ экспоненциально зависит от напряженности электрического поля $F$ и энергии разрыва химических связей в макроионе $D^{+}[28,29]$ :

$$
k_{t f}=\tau_{0}^{-1} \exp \left(-\frac{D^{+}-e l_{0} F}{k_{B} T}\right) .
$$

Здесь $e-$ заряд электрона, $l_{0}$ - активационная длина, $k_{B}-$ постоянная Больцмана, $T-$ температура, $\tau_{0}=10^{-13} \mathrm{~s}$.

В ионизированной макромолекуле энергия разрыва химических межатомных связей понижена. Так, например, энергия разрыва C-C-связи в молекуле этана равна $3.6 \mathrm{eV}$, а в положительном ионе этана $-1.9 \mathrm{eV}$ [30]. В ионе пропионового ангидрида энергия центральной
$\mathrm{C}-\mathrm{C}$-связи составляет $2.4 \mathrm{eV}$, в то время как в нейтральной молекуле пропионового ангидрида $\mathrm{CH}_{3} \mathrm{CH}_{2} \mathrm{CHO}$, энергия этой связи равна $2.9 \mathrm{eV}$ [28] (в ионе этой молекулы заряд делокализован по всем его связям).

В рассматриваемой задаче предполагался ланжевеновский механизм рекомбинации. Однако известно, что в электрическом поле рекомбинация зарядов заторможена, поскольку вероятность разделения пары зарядов экспоненциально быстро возрастает при увеличении напряженности электрического поля. В соответствие с данными работы [31] можно принять, что в полях с напряженностью, превышающей $10^{6} \mathrm{~V} / \mathrm{cm}$ рекомбинация отсутствует, то есть принять, что

$$
k_{r}(t)=\left\{\begin{array}{lll}
\frac{e \mu_{h}}{\varepsilon \varepsilon_{0}}, & \text { если } & F(t) \leq 10^{6} \mathrm{~V} / \mathrm{cm}, \\
0, & \text { если } & F(t)>10^{6} \mathrm{~V} / \mathrm{cm}
\end{array}\right.
$$

где $\mu_{h}$ - подвижность дырок, $\mu_{h}>\mu_{e}$.

Ясно, что при условии $F_{0} \gg F_{a}$ рекомбинация происходит лишь в течение малой доли периода изменения напряжения и поэтому „рекомбинационные“ члены в уравнениях (3)-(5) слабо влияют на результаты расчетов.

Характер зависимостей констант скоростей туннельных электронных переходов $k_{\mathrm{HL}}$ и $k_{\mathrm{HT}}$ от высоты и ширины соответствующих барьеров и напряженности электрического поля приведены в $[8,9]$. При этом снижение энергии ионизации молекул $\Delta I$ при увеличении концентрации зарядов в $[8,9]$ определялось в дебаевском приближении, которое справедливо при сравнительно небольших концентрациях зарядов, то есть в случае идеальной плазмы. Для неидеальной или коллективной плазмы, при повышенных концентрациях зарядов, для определения $\Delta I$ используются другие модели, учитывающие взаимодействие частиц плазмы. Так, например, для описания свойств неидеальной плазмы используется соотношение [10]:

$$
\Delta I=k_{B} T \frac{l}{r_{D}+\Lambda / 8} .
$$

Здесь $r_{D}$ - радиус Дебая, определяемый как

$$
r_{D}=\sqrt{\frac{\varepsilon \varepsilon_{0} k_{B} T}{e^{2} n}}
$$

где $n$ - концентрация зарядов в твердотельной плазме, определяемая в настоящей работе как полная концентрация дырок, равная $n=n_{\mathrm{HL}}^{+}+n_{\mathrm{HT}}^{+}+n_{r}^{+}$,

$$
l=\frac{e^{2}}{4 \pi \varepsilon \varepsilon_{0} k_{B} T}
$$

и

$$
\Lambda=\frac{h}{\sqrt{2 \pi m k_{B} T}},
$$

где $h$ - постоянная Планка, $m$ - масса электрона. 


\section{3. Результаты расчетов и их обсуждение}

Решение системы (3)-(7) позволяет определить изменение концентрации зарядов со временем (предполагается, что концентрация дырок равна концентрации электронов) и время жизни полимера в электрическом поле (долговечность $\tau$ ), если принять, что долговечность совпадает со временем накопления критической концентрации носителей зарядов $n_{\mathrm{cr}}$, после достижения которой скорость накопления зарядов резко увеличивается $[8,9]$.

При проведении подобных расчетов в случае переменного поля принималось, что деградация полимера в положительные и отрицательные полупериоды изменения поля протекает с одинаковой скоростью, то есть при решении системы уравнений (3)-(7), полагалось, что $F(t)=F_{0}|\sin (2 \pi f t)|$, а в качестве начальных условий при $t=0$, принималось: $n_{\mathrm{HL}}^{+}(0)=n_{\mathrm{HT}}^{+}(0)=n_{r}^{+}(0)$ $=N_{t}^{\prime}(0)=0$ и $N_{t}(0)=10^{15} \mathrm{~cm}^{-3}$ (начальная концентрация дырок).

Расчеты проводились при следующих значениях параметров $T=300 \mathrm{~K}, D^{+}=1.8 \mathrm{eV}, l_{0}=0.2 \mathrm{~nm} \mathrm{[28], \varepsilon}=3$, $N_{0}=10^{21} \mathrm{~cm}^{-3}, E_{g}=E_{\mathrm{HOMO}}-E_{\mathrm{LUMO}}=5 \mathrm{eV}\left(E_{\text {НОмо и }}\right.$ $E_{\mathrm{LUMO}}$ - энергии уровней HOMO и LUMO соответственно), $E_{t}=1 \mathrm{eV}, \sigma_{t}=10^{-14} \mathrm{~cm}^{2}, \mu_{h}=10^{-9} \mathrm{~cm}^{2} /(\mathrm{V} \cdot \mathrm{s})$ и $\mu_{e}=10^{-11} \mathrm{~cm}^{2} /(\mathrm{V} \cdot \mathrm{s})$. При $f=50 \mathrm{~Hz}$ значение $d_{0}$, рассчитанное по формуле (1), при $F_{0}=10^{7} \mathrm{~V} / \mathrm{cm}$ составляет $\sim 1 \mu \mathrm{m}$. Учитывалось, что испускает свет только та часть образца, которая соответствует области повышенной напряженности поля в образце, например, вблизи неоднородностей на электродах. При использовании пленочных образцов площадью $\sim 1 \mathrm{~cm}^{2}$ область свечения составит $\sim 1 \%$ от рассматриваемой площади. Поэтому светящийся объем $V_{r}=d_{0} S_{r} \approx 10^{-6} \mathrm{~cm}^{3}$.

Также как и в $[8]$ полагалось, что барьеры имеют треугольную форму, а снижение энергии ионизации $\Delta I$ рассчитывалось по формуле (9).

Типичный вид расчетных зависимостей $n_{\mathrm{HL}}^{+}(t)$ и $n_{\mathrm{HT}}^{+}(t)$ и полной концентрации дырок $n(t)$ представлен на рис. 1. Расчеты показали, что при всех значениях $F_{0}$, в любой момент времени $n_{r}^{+}(t) \ll n_{\mathrm{HL}}^{+}(t), n_{\mathrm{HT}}^{+}(t)$, то есть $n \approx n_{\mathrm{HL}}^{+}+n_{\mathrm{HT}}^{+}$. Из рисунка видно, что на начальном (относительно коротком) этапе процесса старения полимерного диэлектрика основной вклад в накопление зарядов вносят переходы HOMO-LUMO, инициирующие появление ионов $n_{\mathrm{HL}}^{+}$и электронов. Однако со временем концентрация ловушек возрастает до такой степени, что переходы HOMO-TRAP начинают доминировать и в дальнейшем концентрация дырок в плазме будет в основном определяться концентрацией $n_{\mathrm{HT}}^{+}$. Момент пробоя $t_{\mathrm{br}}$ определялся нами по резкому возрастанию концентрации дырок, когда $n_{\mathrm{HT}}^{+}$достигало значения $\sim 10^{19} \mathrm{~cm}^{-3}$. Таким образом, долговечность полимера в переменном электрическом поле $(\tau)$ определяется как время накопления критической концентрации зарядов $n_{\text {cr, }}$, при достижении которой начинается резкий

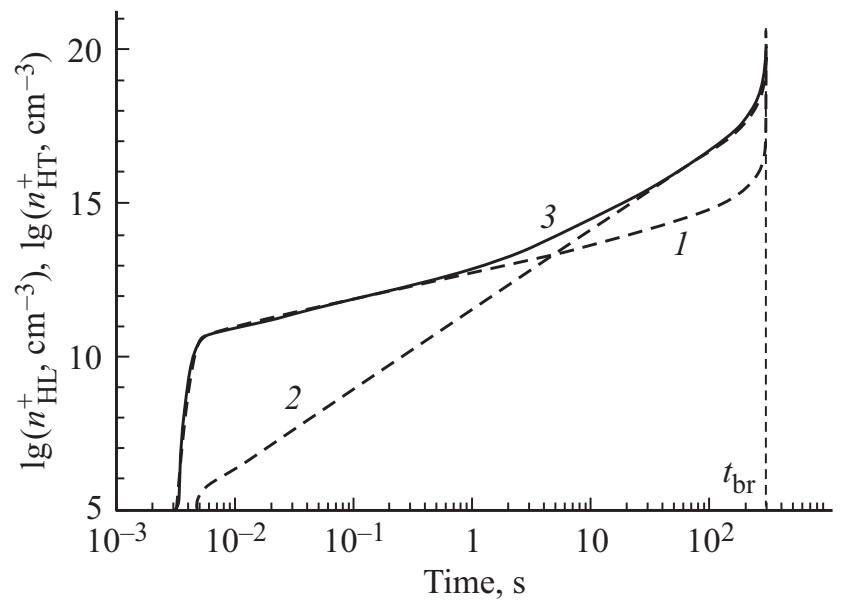

Рис. 1. Зависимости от времени концентраций дырок $n_{\mathrm{HL}}^{+}$ (кривая 1) и $n_{\mathrm{HT}}^{+}$(кривая 2) и полной концентрации дырок $n$ (кривая 3). Расчеты выполнены при $F_{0}=1.4 \cdot 10^{7} \mathrm{~V} / \mathrm{cm}$.

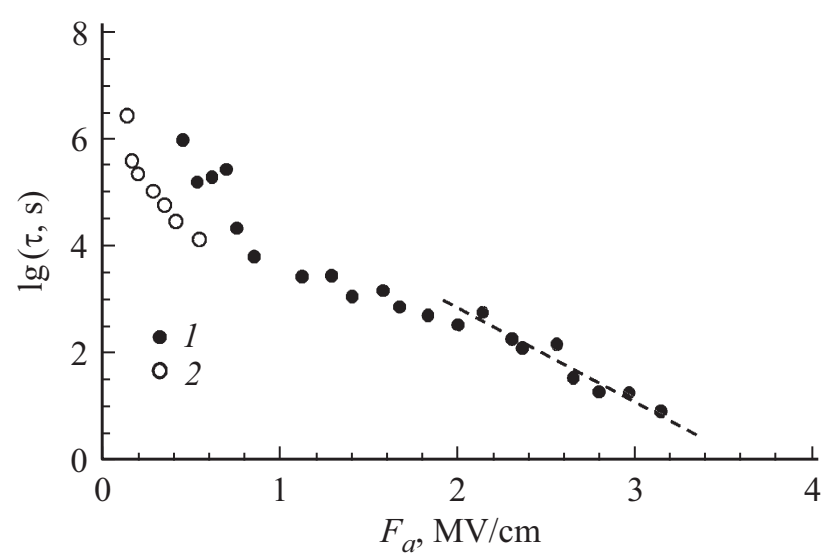

Рис. 2. Сопоставление расчетных (пунктирная прямая) и экспериментальных зависимостей $\tau\left(F_{0}\right)$ для пленок полиэтилентерефталата. $1-[12], 2-[13]$.

рост концентрации зарядов и соответствующее резкое возрастание плотности тока, то есть происходит пробой диэлектрика.

Рассматривая зависимость $n(t)$ при разных значениях $F_{0}$ можно получить зависимости $\tau\left(F_{0}\right)$. При расчетах значения $F_{0}$ выбирались так, чтобы полученные расчетные величины долговечности соответствовали величинам долговечности, регистрируемым в экспериментах.

На рис. 2 пунктирной линией изображены результаты расчета долговечности в зависимости от амплитудного значения переменного электрического поля $(f=50 \mathrm{~Hz})$ при температуре $300 \mathrm{~K}$. На этом же рисунке темными и светлыми кружками показаны экспериментальные данные, характеризующие зависимость долговечности полиэтилентерефталата от амплитудного значения переменного поля этой же частоты, взятые из работ $[12,13]$. При изображении данных работы [13] (светлые кружки) учитывалось, что используемые в этой работе средне- 
квадратичные значения переменного поля (rms) в $\sqrt{2}$ меньше амплитудного значения переменного поля.

При сопоставлении теоретических величин долговечности с экспериментальными данными необходимо учитывать, что действующее в полимерном диэлектрике поле неоднородно. Имеются области усиления поля, например, вблизи неоднородностей на электродах. В этих областях и развиваются процессы разрушения диэлектрика. Пробой полимеров локализованное явление, характеризующееся образованием узкого канала пробоя. На последней стадии пробоя вследствие выделения джоулева тепла при протекании тока высокой плотности образуется область деградации полимерного материала диаметром в несколько микрометров. Оказалось, что расчетные значения долговечности удовлетворительно согласуются с представленными на рис. 2 экспериментальными значениями при величинах $q$, изменяющихся при изменении напряженности поля в диапазоне 3-5. Так при $F_{0}=1.65 \cdot 10^{7} \mathrm{~V} / \mathrm{cm}$ величина $q$ составила 3.90 , при $F_{0}=1.40 \cdot 10^{7} \mathrm{~V} / \mathrm{cm}-q=4.05$, а при $F_{0}=1.38 \cdot 10^{7} \mathrm{~V} / \mathrm{cm}-q=4.89$. Тот факт, что величина $q$ падает с увеличением напряженности поля, свидетельствует, по-видимому, о влиянии объемного заряда (O3) на величину $q$ [32]. О3 эффективно накапливается при увеличении напряженности поля в результате чего снижается напряженность вблизи неоднородностей на электродах. Величина $q=3-5$ представляется вполне разумной и физически оправданной.

Таким образом, предложенный механизм разрушения полимеров в переменном электрическом поле позволил непротиворечиво объяснить имеющиеся в литературе данные об электрической долговечности полимеров в переменном поле.

\section{4. Заключение}

С позиций ионизационного механизма разрушения полимеров в электрическом поле нашло объяснение резкое различие электропрочностных свойств полимеров в постоянном и переменном электрических полях. Показано, что уменьшение долговечности полимеров в переменном поле обусловлено деструкционными процессами, инициированными рекомбинацией инжектированных из электродов носителей зарядов, сопровождающейся выделением в каждом акте рекомбинации энергии в несколько электронвольт. Безызлучательная релаксация электронных возбужденных состояний (триплетных экситонов), вызывающая разрывы связей в макромолекулах с образованием свободных радикалов приводит к образованию химических дефектов (электронных ловушек) в макромолекулах. В результате увеличивается скорость ионизации макромолекул, то есть скорость образования носителей заряда, и ухудшаются электропрочностные свойства полимеров и прежде всего снижается время их жизни в переменном электрическом поле по сравнению с долговечностью полимеров в постоянном электрическом поле.

\section{Конфликт интересов}

Авторы заявляют, что у них нет конфликта интересов.

\section{Список литературы}

[1] L.A. Dissado, J.C. Fothergill. Electrical Degradation and Breakdown in Polymers. Peter Peregrinus, London. (1992). $589 \mathrm{pp}$.

[2] J. Shibuya, S. Zoledovski, J.N. Colderwood. IEEE Trans. Power Apparat. Syst. 96, 198 (1977). DOI: 10.1109/T-PAS.1977.32324

[3] N.R. Tu, K.C. Kao. J. Appl. Phys. 85, 7267 (1999). DOI: $10.1063 / 1.370543$

[4] D. Liufu, X.S. Wang, D.M. Tu, K.C. Kao. J. Appl. Phys. 83, 2210 (1998). DOI: 10.1063/1.366958

[5] Z. Li, Y. Yin, X. Wang, D.M. Tu, K.C. Kao. J. Appl. Polym. Sci. 89, 3416 (2003). DOI: 10.1002/app.12511

[6] П.П. Кобеко. Аморфные вещества. Изд-во АН СССР, М.-Л. (1952). $431 \mathrm{c.}$

[7] J.P. Jones, J.P. Llewellyn, T.J. Lewis. IEEE Trans. Dielectr. Electr. Insul. 12, 951 (2005). DOI: $10.1109 /$ TDEI.2005.1522189

[8] В.А. Закревский, Н.Т. Сударь. ФТТ 55, 1298 (2013).

[9] V.A. Zakrevskii, V.A. Pakhotin, N.T. Sudar. J. Appl. Phys. 85, 234101 (2014). DOI: 10.1063/1.4883365

[10] В. Эбелинг, В. Крефт, Д. Кремп. Теория связанных состояний и ионизационного равновесия в плазме и твердом теле.Мир, М. (1979).

[11] Abdelkrim Laifaoui, Mohend Seghir Herzine, Youcef Zebboudj, Jean-Michel Reboul, Mohammed Nedjar. IEEE Trans. Dielectr. Electr. Insul. 21, 2267 (2014). DOI: 10.1109/TDEI.2014.004190

[12] В.Б. Бережанский, В.М. Быков, В.В. Городов, В.А. Закревский, А.И. Слуцкер. Высокомолекуляр. соединения, А 28, 2163 (1986).

[13] G. Mazzanti, G.C. Montanari, L.A. Dissado. IEEE Trans. Dielec. Electr. Insul. 12, 876 (2005). DOI: 10.1109/TDEI.2005.1522183

[14] C. Laurent, G. Teyssedre, G.C. Montanari. IEEE Trans. Dielectr. Electr. Insul. 11, 554 (2004). DOI: 10.1109/TDEI.2004.1324344

[15] J.M. Alison, J.V. Champion, S.J. Dodd, G.C. Stevens. J. Phys. D 28, 1693 (1995).

[16] F. Baudoin, D.H. Mills, P.L. Lewin, S. Le Roy, G. Teyssedre, C. Lauren. J. Phys. D 44, 165402 (2011). DOI: 10.1088/00223727/44/16/1654

[17] B. Qiao, G. Teyssedre, C. Laurent. J. Appl. Phys. 119, 024103 (2016). DOI: 10.1063/1.4939824

[18] T. Lebey, C. Laurent. J. Appl. Phys. 68, 275 (1990). DOI: $10.1063 / 1.347197$

[19] C. Laurent, F. Massines, C. Mayoux. IEEE Trans. Dielectr. Electr. Insul. 4, 585 (1997). DOI: 10.1109/94.625646

[20] G. Teyssedre, G. Tardieu, D. Mary, C. Laurent. J. Phys. D 34, 2220 (2001). DOI: JPhysD/34/2220

[21] L.E. Lyons. J. Chem. Phys. 5001 (1957).

[22] В.К. Милинчук, Э.Р. Клиншпонт, С.Я. Пшежецкий. Макрорадикалы. Химия, М. (1980). 264 с. 
[23] Б. Ранби, Я. Рабек. Фотодеструкция, фотоокисление, фотостабилизация полимеров. Мир, М. (1978).

[24] Е.Ю. Астахов, Э.Р. Клиншпонт, В.К. Милинчук. ХВЭ. 27, 29 (1993).

[25] В.А. Закревский, В.Е. Корсуков. Высокомолекуляр. соединения. А 14, 955 (1972).

[26] S. Le Roy, G. Teyssedre, C. Laurent. IEEE Trans.Dielectr. Electr. Insul. 4, 644 (2005). DOI: 10.1109/TDEI.2005.1511090

[27] D. Mary, M. Albertini, C. Laurent. J. Phys. D 30, 171 (1997).

[28] Э.Н. Король, В.В. Лобанов, В.А. Назаренко, В.А. Покровский. Физические основы полевой масс-спектроскопии. Наук. думка, Киев (1978). 192 с.

[29] V.A. Zakrevskii, N.T. Sudar, A. Zaopo, Y.A. Dubitsky. J. Appl. Phys. 93, 2135 (2003). DOI: 10.1063/1.1531820

[30] R.A.W. Johnstone. Mass Spectrometry for Organic Chemists. Cambridge University, Cambridge (1972).

[31] А.П. Тютнев, В.С. Саенко, Е.Д. Пожидаев, Н.С. Костюков. Диэлектрические свойства полимеров в полях ионизирующих излучений. Наука, М. (2005). 453 с.

[32] В.А. Закревский, Н.Т. Сударь. ЖТФ 60, 66 (1990).

Редактор Т.Н. Василевская 